Artigo

\title{
El lenguaje como factor de desigualdad en los aprendizajes en Pisa 2009 El caso de la frontera noreste de Uruguay con Brasil
}

\author{
Language as an inequality factor in learning in Pisa 2009 \\ A case study at the boundary between Uruguay and Brazil

Tabaré Fernández*
Angela Ríos**
Agustina Marques***

Resumen: La frontera de Uruguay con Brasil ha sido históricamente identificada como la región con los peores indicadores socioeconómicos del país. Por otro lado, la frontera es una región bilingüe, con una situación considerada desde el campo de los estudios lingüísticos como de diglosia. Esto nos hace cuestionar si las diferencias en los resultados educativos de esta región tienen que ver específicamente con el uso del "portuñol" por los estudiantes que viven en la fontera. El objetivo de esta investigación es analizar el efecto aislado de ser un hablante de "portuñol" y vivir en la frontera, incluyendo además de los determinantes educativos clásicos, factores lingüísticos, a partir de los microdatos de Pisa 2009. Los resultados permiten confirmar la hipótesis de una desigualdad lingüística en el nivel individual.

Palabras clave: Desigualdad educativa. Factores lingüísticos. Frontera.

\begin{abstract}
The Uruguayan borderline with Brazil has historically been identified as a region with the worst socioeconomic indicators of the country. On the other side, Uruguayan borderline with Brazil is a bilingual region, with a categorized situation of diglossia from the earliest linguistic studies. This makes us wonder if differences on educational results are related with the use of "portuñol" on behalf of the Uruguayan borderline students. The aim of this research is to analyze the isolated effect of being a "portuñol" speaker and live in the borderline, over a standard model of educational inequality (social class, gender, type of educational institution). In order to achieve this we generated a Hierarchical Linear Model in two levels: students and schools, adding to the classical educational determinants linguistic factors, using microdata of Pisa 2009. The results allow us to confirm the hypothesis of a linguistic inequality on an individual level.
\end{abstract}

Keywords: Education inequality. Linguistic factors. Boundaries.

\footnotetext{
*Doctor en Sociología por el Colegio de México (Colmex, México, México D.F.), profesor de Sociología en la Facultad de Ciencias Sociales, Montevideo, Uruguay<tfaguerre@gmail.com>. ** Candidata a Master in Science en Demografía por la Facultad de Ciencias Sociales (FCS, UdelaR, Montevideo, Uruguay) <angelariosgonzalez@gmail.com>.

*** Candidata a Master in Science en Sociología por la Facultad de Ciencias Sociales (FCS, UdelaR, Montevideo, Uruguay), profesora ayudante de investigación en CCI UdelaR, Montevideo, Uruguay<amarques@cci.edu.uy>.
} 


\section{Problema y objetivos}

El examen de la desigualdad educativa en la educación básica de Uruguay tiene una formulación estándar de consenso que incluye cuatro factores: i) el origen social (en términos de clase social o nivel socioeconómico); ii) el sexo del estudiante; iii) el entorno sociocultural de la escuela; y iv) el sector institucional (Cervini, 2002; Fernández, 2003; Fernández, 2007b; Fernández, 2007a; Blanco, 2011). La formulación así planteada es consistente con una teoría que define la desigualdad educativa en términos multinivel donde a nivel individual opera una estratificación social de clase y género iniciada en la socialización familiar que tanto se apoya en, así como interactúa con, una base macrosocial de segmentación social y diferenciación institucional (Fernández, 2010). Al extender este modelo a la educación media, se ha hecho imprescindible considerar además de los anteriores, las diferencias entre las modalidades curriculares: educación general y educación técnica (Cervini, Basualdo, 2003; Fernández, et al., 2007).

En varios países de la región, tales como Brasil, Ecuador, Guatemala, México, Paraguay o Perú, el modelo básico se considera incompleto si no se integran factores relacionados con la lengua, la etnia o la raza. Si bien estos conceptos delimitan situaciones sociales bien diferentes por razones históricas, lingüísticas y sociales, existe un elemento que asemeja a todas: se trata de grupos sociales triplemente marginados: i) porque se comunican en una lengua que no es socialmente dominante; ii) porque residen en zonas rurales frecuentemente aisladas; y iii) por las condiciones de pobreza estructural en la que han vivido generación tras generación (Gonzalbo, 1999; Pérez, 2003). En todos estos casos, la estimación de modelos extendidos de desigualdad entrega resultados estadísticamente significativos (Ortiz, 2012; Parker, Rubalcava, Teruel, 2003). Si bien la interpretación de los resultados no ha sido consensuada, en particular, la proposición de mecanismos explicativos, las implicancias para la política han ingresado ampliamente en el debate político, tanto en términos de la educación como los derechos humanos (Stavenghagen, 1998).

El objetivo general de este trabajo es realizar una aproximación al tema del lenguaje como factor de desigualdad educativa en Uruguay, estimando tres modelos de regresión jerárquico lineales (HLM) que evalúen la hipótesis más general sobre la incidencia marginal del portuñol sobre los resultados de los estudiantes de Educación Media en el desarrollo de su competencia lingüística en el español. Se utilizarán los microdatos de la única evaluación de aprendizajes que hasta el presente ha incluido indicadores para identificar a los usuarios del portuñol: Pisa 2009. 


\section{Antecedentes}

Prácticamente en todos los países de la región se ha verificado una creciente sensibilidad y aceptación académica de la tesis de que la desigualdad lingüística tiene un efecto marginal, neto significativo y sustantivo que se diferencia de los efectos generados por la marginación histórica de los pueblos indígenas, de la ruralidad o de las políticas raciales. Esto ha llevado a una creciente reivindicación primero y luego reformas que han hecho visible la lesión de derechos de quienes no hablan desde la infancia la lengua de uso oficial en el ámbito educativo. Se ha legislado constitucionalizado la igualdad de derechos lingüísticos o legislado contra la discriminación étnico/lingüística, se ha modificado los currícula o se reformó o se creó programas de educación bilingües.

Uruguay no escapó a esta "ola de reconocimientos y reformas". La ley general de educación, no 18347 de diciembre de 2007 modificó el marco legal monolingüe que implícita o explícitamente había imperado en el país desde sus inicios en algunas etapas con especial agresividad, manteniendo un discurso purista del lenguaje y juzgando como negativa la "contaminación" de las lenguas. En el artículo 40 se establecen las líneas transversales, entre las cuales se introduce la "educación lingüística", noción que se define en el numeral 5) como

el desarrollo de las competencias comunicativas de las personas, el dominio de la lengua escrita, el respeto a las variedades lingüísticas, la reflexión sobre la lengua, la consideración de las diferentes lenguas maternas existentes en el país (español del Uruguay, portugués del Uruguay, lengua de señas uruguaya) y la formación plurilingüe a través de la enseñanza de segundas lenguas y lenguas extranjeras.

Los alcances no fueron reglamentados con precisión, pero contribuyeron a legitimar las nuevas políticas lingüísticas iniciadas desde el 2003 con el programa de Educación Bilingüe para la Educación Primaria (español-inglés y luego español-portugués) e impulsadas decididamente desde el 2005 por el gobierno de izquierda del Frente Amplio (Broveto, 2010).

El principal avance que esta novedad jurídica trajo, desde el punto de vista de las políticas educativas al menos, ha sido la legitimación oficial del idioma "portugués del Uruguay", "dialecto portugués del Uruguay", "portugués de frontera" o más coloquialmente "portuñol".

Como se expresó anteriormente, en muchos países de la región se dio una diglosia, es decir lenguas en contacto con jerarquías, entre el idioma estándar y 
el de los pueblos originarios. (Ortiz, 2012). En la zona fronteriza de Uruguay con Brasil, esto sucedió entre el español, idioma de los centros educativos, y el llamado portugués del Uruguay, fronterizo o portuñol. En el 1995 en el ámbito del Mercosur se elevó al portugués a la altura del español, nombrándolos a ambos idiomas oficiales, implementando el bilingüismo. Esto implicó la enseñanza de portugués estándar, no del mencionado portuñol, lo que implicó una nueva diglosia, con la lengua fronteriza sometida ahora a dos lenguas estándar. En el ámbito de la práctica esto tuvo implicancias fundamentales ya que sólo la población español monolingüe utilizó en la escuela un idioma coincidente con el de su ámbito privado o doméstico (Barrios, 2001; Ortiz, 2012).

Diferentes estudios realizados establecen que el "portugués del Uruguay", "dialecto portugués del Uruguay", "portugués de frontera" o más coloquialmente "portuñol" se trata de una lengua variedad del portugués, no estandarizada. Se da en territorio uruguayo pero es evidentemente un lengua derivada del portugués, influenciada por el contacto con el español (Barrios et al. 1993). Durante casi todo el siglo 20 ha sido la lengua socialmente predominante en los intercambios comerciales y en las interacciones cotidianas en la frontera, sumándosele desde mediados de los setenta, a la lengua principal en la oferta de televisión (aérea o por cable) a la que acceden los habitantes uruguayos de las localidades fronterizas, teniendo incluso gran presencia en los programas de radios locales (tanto AM como FM). Su generalización tiene relación con la fuerte presencia de propietarios rurales brasileños presentes desde la finalización de la Guerra Grande (1852) y contemporáneamente también con la presencia de trabajadores rurales brasileños. También los estudios informan que se trató de una lengua socialmente diferenciada, asociada a las clases trabajadoras manuales, y reservada al plano doméstico (Behares y Elizaincín, 1981; Elizaincín, Behares y Barrios, 1987; Elizaincín 1992). Es una lengua proscripta de la escuela (tanto primaria como media) y también de los distintos procedimientos que los ciudadanos tengan que hacer por escrito ante el estado (Bertolotti y Coll, 2014).

En los últimos diez o quince años podríamos afirmar que es perceptible una tendencia cada vez más nítida hacia la des/estigmatización del uso del dialecto, al punto que en 2015 comenzó una campaña en la frontera para realizar una petición ante la Unesco de protección cultural del portuñol. ${ }^{1}$ Nosotros adoptamos el criterio de llamarle "portuñol" al objeto lingüístico

\footnotetext{
${ }^{1}$ Vease por ejemplo <http://cultura.elpais.com/cultura/2015/07/23/actualidad/1437685636_ 246770.html>.
} 
"dialectos portugueses de la frontera de Uruguay con Brasil". ${ }^{2}$ En particular, estos cambios han permitido generar un espacio innovador donde el portuñol se ha integrado como lengua legitimada a nivel escolar, sea como "segunda lengua" o "lengua equivalente en el aula". Esto implica un giro completo desde la Reforma de José Pedro Varela de 1877 hasta la Reforma de Rama (19962000) en el cual la política lingüística en la escuela fue enseñar la variedad estándar del español (de Uruguay) y restringir o directamente censurar el uso del portuñol (Anep, 2008; Bertolotti, Coll, 2014; Behares, 2007).

Ahora bien, este avance en el campo de los derechos tuvo particularidades e inconsistencias. Por un lado, el bilingüismo entre lenguas maternas quedó restringido a la frontera: es excepcional el portuñol en las escuelas o secundarios del litoral, del centro del suroeste o del área metropolitana de Montevideo.

Por otro lado, la nueva línea en la política no generó una base empírica apropiada. Mencionaremos algunas paradojas a título de ejemplo. El Censo de Población de 2011 no incluyó ninguna pregunta que permitiera conocer cuál es la lengua materna de los habitantes del país, ni cuáles son las otras lenguas que habla, por lo que el país sigue sin tener un parámetro censal sobre cuál es la proporción de hablantes del portuñol. Las evaluaciones nacionales de aprendizaje en 6tos de Primaria realizadas en el país (1996, 1998, 1999, 2002, 2005, 2009 y 2013) no incluyeron ninguna variable que permitiera inferir cual era la lengua materna del alumno. Uruguay tampoco incluyó identificación lingüística alguna en Pisa (2003, 2006, 2012 ni 2015), ni en el Tercer Estudio Regional Comparativo y Explicativo (Terce) de la Unesco en América Latina. En el caso particular del Programa Pisa, el país participó con instrumentos en idioma español, lo que implica declarar que la proporción de hablantes de otras lenguas era menor al 5\%. La única ocasión que una evaluación de aprendizajes tuvo variables de identificación lingüística fue en el ciclo 2009 de Pisa, en el cual además, se sobre representó la frontera con Brasil a los efectos de llevar adelante estudios específicos.

\section{Método y datos}

\section{Delimitación geográfica y lingüística}

Siguiendo a Cánepa y Carreño (2011), la región de "frontera" ha sido definida como área geográfica amplia, contigua a la frontera con Brasil, que

\footnotetext{
${ }^{2}$ Con este criterio no solo pretendemos recuperar positivamente el término, sino que entendemos que es una nominación subjetiva local que permite además distinguirla de otras variedades fronterizas del portugués que se han desarrollado en los restantes seis países con los que Brasil tiene frontera.
} 
cubre la totalidad de los departamentos de Rivera y Cerro Largo, la zona este del departamento de Artigas, así como el norte del departamento de Tacuarembó. ${ }^{3}$ Comprende dos redes de localidades interconectadas vialmente: una red sobre las ruta 5, 30, 27 y 29 ; en tanto que la otra, se erige sobre las rutas 7,8 y 27. Entre sí están unidas principalmente por las rutas 26 al sur y la ruta 27 limítrofe. Este territorio cubre el $10 \%$ de la superficie nacional y tiene una población de 365 mil habitantes (el 11\%) según resultados del Censo de población de 2011.

Esquema 1. Mapa con la distribución estimada actual del Portugués del Uruguay o portuñol en el territorio uruguayo

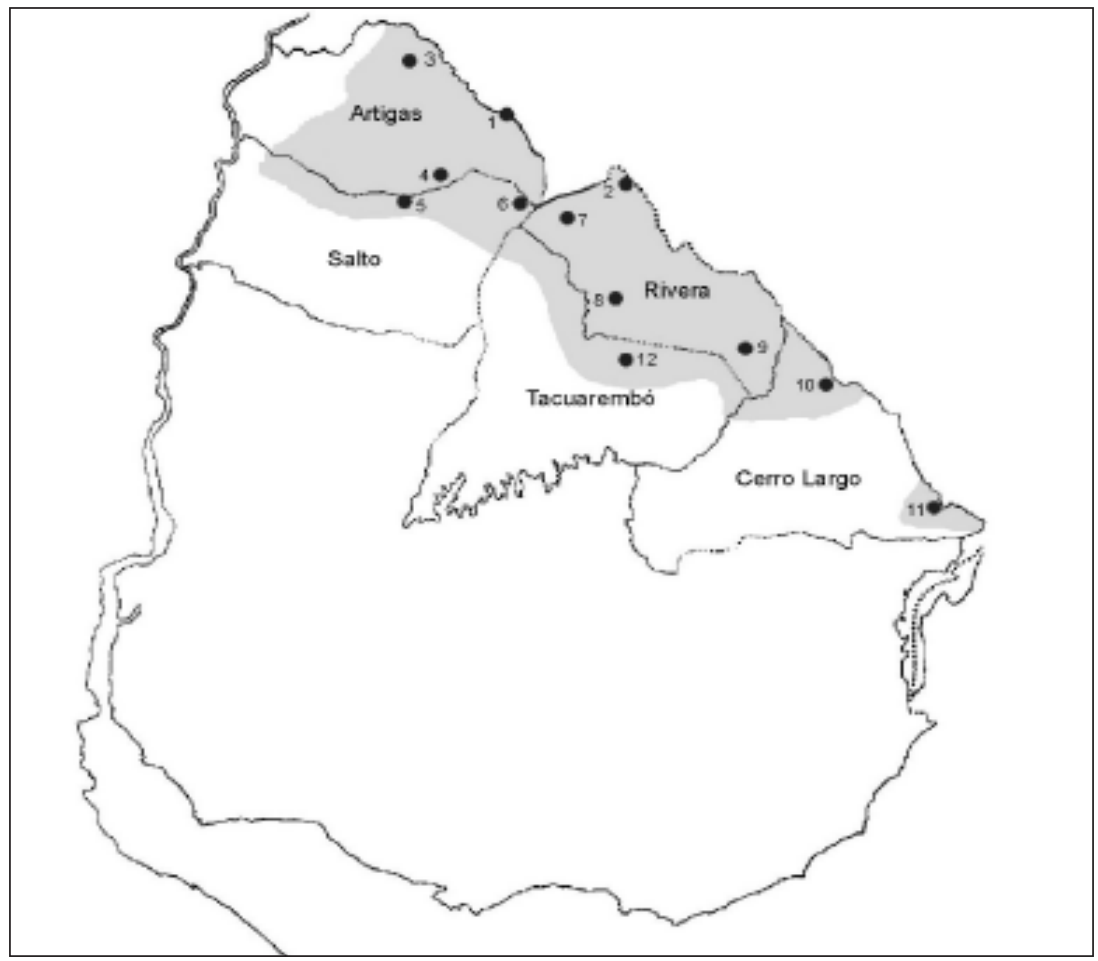

Fonte: Behares (2007). Referencias: 1. Artigas; 2. Rivera; 3. Bernabé Rivera; 4. Sequeira; 5. Colonia Lavalleja; 6. Masoller; 7. Tranqueras; 8. Minas de Corrales; 9. Vichadero; 10. Aceguá-Noblía; 11. Río Branco.

${ }^{3}$ Secciones censales 1, 2, 3 y 4 de Artigas donde están la capital y las localidades de Bernabé Rivera, Sequeira, Cuaró, Pintadito. Secciones censales 1, 2, 4, 5 y 14 del departamento de Tacuarembó donde además de la capital, están las localidades de Ansina, Curtina y Tambores. Se incluye aquí todo el departamento de Rivera y de Cerro Largo. 
Este territorio de frontera es más extenso que la zona geográfica del portuñol que ha sido propuesta por Behares (2007) sobre Rona (1965). Los estudios dialectológicos informan por ejemplo que el portuñol no tendría una presencia importante ni en la ciudad de Melo ni en la ciudad de Tacuarembó, por lo que ambas áreas urbanas deberían ser excluidas (Ver esquema 1 con las anotaciones). A su vez, la zona noreste del departamento de Salto debiera estar contemplada en esta área geográfica. En este trabajo y dada la especificidad del tema lingüístico, adoptamos esta definición más restringida de "frontera".

\section{Muestra}

Una delimitación muy similar a la definición de frontera como región bilingüe (pero sin incluir Colonia Lavalleja y el noreste del departamento de Salto) fue adoptada por el Programa Pisa de Uruguay para diseñar la muestra nacional que participara en el Ciclo 2009. Ésta incluyó el área del portugués del Uruguay como una variable para la estratificación. La tabla 1 muestra la distribución estimada de centros educativos y estudiantes de 15 años en el universo (2008) y en la muestra seleccionada para Pisa 2009. Vale añadir un aspecto importante respecto a la cobertura. En 2009, Pisa evaluó a los jóvenes escolarizados en un centro de educación formal del nivel medio, púbico o privado, nacidos entre el 1 de abril de 1993 y el 31 de marzo de 1994. A nivel nacional, el 82\% de los jóvenes de 15 años estaban escolarizados en el año 2009 en un centro formal de estas características. En la región de frontera así definida, la tasa de escolarización (y por tanto el universo al cual se pueden inferir los hallazgos de esta investigación) era más baja que a nivel nacional y alcanzó al $75 \%$ de la cohorte de aquella edad. Esta es una característica persistente y configura un elemento central de la singularidad regional desde el punto de vista de la política educativa (Fernández y Ríos, 2013).

Tabla 1. Distribución de centros educativos y estudiantes de 15 años en la zona del portuñol en Uruguay, universo registrado en la Educación Pública según fuentes administrativas de 2008 y la muestra nacional efectivamente realizada para Pisa 2009.

Entre paréntesis porcentajes ponderados sobre el total nacional.

\begin{tabular}{lcc}
\hline & $\begin{array}{c}\text { Universo } \\
(\mathbf{2 0 0 8})\end{array}$ & $\begin{array}{c}\text { Muestra } \\
(\mathbf{2 0 0 9 )}\end{array}$ \\
\hline Centros Educativos & $37(5.4 \%)$ & $25(5.4 \%)$ \\
Estudiantes de 15 años escolarizados & $2500(7.0 \%)$ & $708(5.4 \%)$ \\
\hline
\end{tabular}

Fuente: Elaboración propia con base en el marco muestral (2008) y los microdatos liberados de Pisa 2009. 


\section{Indicadores}

El set de variables a utilizar para la estimación se presenta en la tabla 2. Cinco provienen del cuestionario aplicado a los jóvenes conjuntamente con la evaluación Pisa 2009. Se identifican estas variables como fuente OECD: están incluidas en la base internacional liberada en el sitio web del ciclo 2009. La identificación de la zona del portuñol proviene del documento de la muestra diseñada por Uruguay, que se identifica en la base internacional con la etiqueta "stratum".

El índice de entorno sociocultural del centro educativo fue elaborado por nosotros con el mismo fundamento conceptual de la metodología Anep pero utilizando el índice de estatus social cultural y económico del estudiante (ESCS) que la OECD incluye en la base internacional. Estadísticamente, es el promedio ponderado del índice para el centro educativo (XESCS). La identificación de la región de frontera se elaboró con base a información provista por la Anep, quien a pedido especial ubicó los centros en las localidades censales y entregó esta variable (region 9). La identificación de los usuarios del portuñol proviene del cuestionario nacional aplicado para Pisa 2009 y es descripta en el apartado siguiente. De esta medida, luego se construyó un indicador agregado para cada centro educativo, con el objetivo de contar con una variable proxy que informara la composición lingüística del centro.

\section{Estudiante hablante del portuñol}

La identificación de los hablantes del portuñol siguió la siguiente metodología. El cuestionario de estudiantes aplicado en 2009 incluyó un set de preguntas específicamente diseñadas por el Programa Pisa Uruguay de la Anep donde se interrogaba al estudiante por los lugares en que hacía "uso del portugués o del portuñol" (Véase esquema 2). La lista de lugares refiere a distintos tipos de interacción según el uso de la lengua (escolar, familiar, comercial, vecinal, grupo de pares) cada uno de los cuales tiene distinta regulación y legitimidad. Aunque estrictamente no son equiparables, todos informan de un uso frecuente, regular y competente desde un punto de vista pragmático. El estudiante podía responder independientemente cada ítem. Las respuestas tienen un alto grado de consistencia: la correlación ítem índice más baja es de 0.61 (la más alta es de 0.83 ) en tanto que el alfa de Cronbach es igual a $0.81 .{ }^{4}$ En consecuencia y con la finalidad de realizar un

\footnotetext{
${ }^{4}$ En análisis factorial confirma que existe una única dimensión con un Eigen value igual a 2.78 que explica el $55.6 \%$ de la varianza estandarizada de los cinco ítemes. El test de Kaiser-MeyerOlkin es igual a 0.822 . Todo esto permite desechar una hipótesis de multidimensionalidad subyacente y hace razonable sostener que más allá de las diferencias de frecuencia en el uso, la escala auto-identifica hablantes del portuñol.
} 
resumen general, optamos por construir un índice simple que identifica como hablante a quien hubiera marcado al menos una de las instancias presentadas. Metodológicamente, la escala tiene como debilidad que se basa en la expresión de la propia conciencia que lleva a la auto-identificación. Esta operación cognitiva y discursiva puede ser inválida en al menos dos contextos. Por un lado, si se pudiera afirmar que existe una proscripción o al menos una fuerte estigmatización social de la lengua minoritaria. Por otro, en un contexto de débil diferenciación de las lenguas. Si bien la primera hipótesis tiene reducido sustento desde fines del siglo 20, la segunda encuentra mayores apoyos empíricos en los estudios de dialectología que se han hecho y que documenta la bibliografía (Bertolotti et al., 2014). En cualquiera de los dos casos, podría achacársele a la medida que estaría subestimando la población de usuarios.

Esquema 2. Definición operativa de los conceptos en la base de datos Pisa 2009.

\begin{tabular}{|c|c|c|c|}
\hline Concepto & Variable & Definición operativa & Fuente \\
\hline \multicolumn{4}{|c|}{ Variables de nivel individuo } \\
\hline Clase social & Escs & $\begin{array}{l}\text { Índice construido por teoría de respuesta al } \\
\text { ítem sobre la base de la educación materna, la } \\
\text { educación paterna, la posesión de ítemes de } \\
\text { confort, posesión de bienes culturales y el más } \\
\text { alto status ocupacional entre padre y madre } \\
\text { en el hogar. Re-escalado con promedio } 0 \text { y } \\
\text { varianza } 1 \text { para Uruguay }\end{array}$ & OECD \\
\hline Género & St04 & Recodificada para que 1 sea mujer y 0 varón & OECD \\
\hline $\begin{array}{l}\text { Hablante de } \\
\text { portuñol }\end{array}$ & $\mathrm{Pu} 2$ & $\begin{array}{l}\text { Índice construido sobre la información } \\
\text { aportada por las preguntas nacionales incluidas } \\
\text { en el cuestionario al estudiante: St } 03 n 02 \text { a } \\
\text { st } 03 \text { n06. }\end{array}$ & ANEP \\
\hline \multicolumn{4}{|c|}{ Variables de nivel escuela } \\
\hline $\begin{array}{l}\text { Sector } \\
\text { institutional: } \\
\text { Privado }\end{array}$ & stratum & $\begin{array}{l}\text { Recodifica para que tome valor } 1 \text { cuando } \\
\text { se trata de una escuela privada y } 0 \text { en los } \\
\text { restantes casos }\end{array}$ & OECD \\
\hline $\begin{array}{l}\text { Sector } \\
\text { institucional: } \\
\text { Técnico }\end{array}$ & stratum & $\begin{array}{l}\text { Recodificada para que tome valor } 1 \text { cuando se } \\
\text { trata de una escuela del Consejo de Educación } \\
\text { Técnica Profesional (UTU) y } \\
0 \text { en los restantes casos }\end{array}$ & OECD \\
\hline $\begin{array}{l}\text { Entorno } \\
\text { sociocultural }\end{array}$ & xescs & $\begin{array}{l}\text { Promedio ponderado para la escuela de la } \\
\text { variable escs }\end{array}$ & $\begin{array}{l}\text { Elaboración } \\
\text { propia }\end{array}$ \\
\hline $\begin{array}{l}\text { Zona del } \\
\text { portuñol }\end{array}$ & Zdpu & $\begin{array}{l}\text { Variable dicotómica que informa si la escuela } \\
\text { se encuentra en la zona del portuñol }\end{array}$ & $\begin{array}{l}\text { Elaboración } \\
\text { propia }\end{array}$ \\
\hline $\begin{array}{l}\text { Composisión } \\
\text { lingüística }\end{array}$ & ppu2 & $\begin{array}{l}\text { Proporción de estudiantes evaluados por Pisa } \\
2009 \text { en la escuela que se auto-identifican } \\
\text { como usuarios del portuñol. }\end{array}$ & $\begin{array}{l}\text { Elaboración } \\
\text { propia }\end{array}$ \\
\hline
\end{tabular}

Fuente: Elaboración propia con base en los microtados Pisa 2009 de Uruguay. 
Esquema 3. Cuestionario nacional aplicado al estudiante en Pisa 2009 con la pregunta de autoidentificación de usuario del portuñol (variable st03n).

\section{P22 Además del español, ¿utilizas portugués o portuñol para comunicarte en algunos de los siguientes ámbitos?}

(Marca todas las opciones que corresponda)
a) No, sólo uso español (Pasa a P23)
Si No
b) Sí, con mi familia
c) Sí, con mis amigos
d) Sí, en el centro educativo fuera de clase
e) Sí, en mis clases en el centro educativo
f) $\mathrm{Si}$, en el barrio (con vecinos, en el comercio, etc.)

Fuente: Cuestionario al estudiante aplicado en Uruguay para el ciclo 2009. Anep.

\section{Método de estimación}

Adoptamos como método de estimación el modelo jerárquico lineal (HLM) (Raudenbush e Bryk, 2002) de dos niveles, estudiantes y escuelas, utilizando los valores plausibles 1 a 5 provistos por la evaluación Pisa, con los ponderadores respectivos. Dado el objetivo exploratorio de este estudio, solo especificamos efectos principales en cada nivel (sin interacciones entre niveles o dentro del mismo nivel), consideramos aleatoria sólo la constante, y nos restringimos sólo a su modelado (multilevel random intercept model). Todas las pendientes de nivel 1 han sido especificadas sin términos aleatorios en el nivel 2.

$$
\text { Ecuación 1: } \quad y_{i j}=\gamma_{00}+\sum_{s=1}^{S} \gamma_{s} w_{s j}+\sum_{q=1}^{Q} \gamma_{q} x_{q j}+u_{0 j}+r_{i j}
$$

Donde y es el puntaje en lectura, $\mathrm{s}=1,2, . ., \mathrm{S}$ son los regresores del nivel escuela, $\mathrm{q}=1,2$, $\mathrm{Q}$ son los regresores del nivel estudiante; $\gamma_{00}$ es la gran media condicional o constante del modelo; $\gamma$ son los coeficientes de regresión estimados; $u_{0}$ es el término aleatorio del nivel escuela $\mathrm{y}_{\mathrm{ij}}$ es el término aleatorio del nivel estudiante. 


\section{Resultados}

\section{Estimación de la población estudiantil usuaria del portuñol}

Los análisis hechos con los microdatos de Pisa informan que si bien sólo un 5.4\% de los jóvenes evaluados residía en la zona geográfica delimitada por el PU, la incidencia de esta lengua es mucho mayor. A nivel nacional, un $16.2 \%$ de los estudiantes evaluados harían uso del PU en cinco tipos de ámbitos públicos y privados por los cuales fueron consultados (véase supra), magnitud que se restringiría al $10.7 \%$ si la operacionalización incluye referencia a ámbitos locales (centro educativos y barrios). La mitad de los jóvenes evaluados en la zona de frontera informaron utilizar con frecuencia el portuñol, mayoritariamente en ámbitos privados, aunque no resulta nada despreciable el porcentaje que es hablante en el centro educativo tanto en el recreo (19.5\%) como en clase (12.7\%). Ahora también es importante anotar un hallazgo no esperado: pero siete de cada diez usuarios frecuentes del PU residirían fuera de la zona de frontera delimitada por Pisa 2009, siendo importante la presencia de hablantes en el litoral, en el centro y en el este del país.

\section{Estimación de los modelos alternativos}

Los resultados de la estimación de los modelos, además del modelo estándar de desigualdad, que se tomará como línea de base de la contribución neta que cada variable aporta a la explicación de la desigualdad, se presentan a continuación en la tabla 2.

Tabla 2. Estimación del porcentaje de usuarios de portuñol en general y en diferentes ámbitos, entre los jóvenes uruguayos evaluados por Pisa 2009 según región.

\begin{tabular}{lcrrrrr}
\hline & $\begin{array}{c}\text { Usuario de } \\
\text { portuñol }\end{array}$ & familia & amigos & CE recreo & CE clase & Barrio \\
\hline MVD + Canelones & $11.6 \%$ & $5.2 \%$ & $4.4 \%$ & $3.4 \%$ & $4.4 \%$ & $2.1 \%$ \\
Suroeste & $7.1 \%$ & $2.8 \%$ & $3.7 \%$ & $3.5 \%$ & $4.3 \%$ & $1.8 \%$ \\
Litoral & $17.3 \%$ & $8.9 \%$ & $7.4 \%$ & $6.6 \%$ & $6.0 \%$ & $5.3 \%$ \\
Frontera Norte & $46.2 \%$ & $27.4 \%$ & $33.0 \%$ & $19.5 \%$ & $12.7 \%$ & $23.5 \%$ \\
Este & $17.6 \%$ & $6.9 \%$ & $10.6 \%$ & $4.9 \%$ & $6.7 \%$ & $5.5 \%$ \\
Central & $12.9 \%$ & $4.3 \%$ & $4.9 \%$ & $3.3 \%$ & $8.3 \%$ & $1.4 \%$ \\
Total & $16.1 \%$ & $7.8 \%$ & $8.2 \%$ & $5.5 \%$ & $5.9 \%$ & $4.9 \%$ \\
\hline
\end{tabular}


Tal como era esperable conforme a la bibliografía consultada, el primer modelo condicional informa que en la zona geográfica del portuñol en promedio y hasta los 15 años, los alumnos han alcanzado un menor desarrollo de la competencia lectora en el español estándar. En esta zona, la magnitud de la brecha frente a cualquier otra región del país alcanza a 56 puntos, lo que en términos estadísticos es altamente significativo: representa más de veinte veces el error estándar de la estimación.

Ahora bien, al contrastar esta brecha con el modelo estándar de la desigualdad que computa la clase social, el género, el entorno sociocultural y el sector institucional, las diferencias se reducen sustantivamente y además pierden significación estadística. Esto es, la introducción de los factores de desigualdad mencionados remueven la casi totalidad del efecto neto geolingüístico y solo queda un componente mínimo cuyo efecto no permite descartar la hipótesis nula.

Distinto es el impacto sobre el nivel de competencia lectora cuando se considera si el estudiante se autoidentifica como usuario del portuñol. El coeficiente es estadísticamente significativo y tiene el signo esperado por hipótesis. El modelo 2 estima que, manteniendo otras condiciones constantes, aquel se reduce en más de 2 desvíos estándares (10 puntos de la prueba). Esto otorga gran plausibilidad a la hipótesis de la interacción lingüística.

A su vez, el modelo 3 al explorar la hipótesis composicional, arroja un coeficiente negativo pero no significativo. Esto contradice la hipótesis de la política. La condición mayoritaria del habla de portuñol no constituiría un beneficio neto para los estudiantes. Dicho de otra forma, el sistema de enseñanza mantiene la desigualdad lingüística a pesar de que los usuarios del sistema sean mayoritariamente hablantes de una lengua que no es el español.

Debe notarse que tiene una magnitud casi despreciable el ajuste del modelo, estimado a partir de los cambios en la proporción de reducción del error de predicción o varianza no explicada (Pseudo $\mathrm{R}^{2}$ ). El modelo estándar explicaría por sí solo un $60.6 \%$ de la varianza, en tanto que la incorporación del uso del portuñol (modelo 3) no aporta sustantivamente a dicha magnitud, a pesar de que el coeficiente es estadísticamente significativo.

\section{Discusión de resultados}

El estudio ha evaluado el efecto neto de ser hablante de portuñol y de estar geográficamente localizado en la frontera, sobre un modelo estándar de desigualdad educativa (clase, género, sector institucional). Los resultados permitieron avalar la hipótesis de la desigualdad lingüística "individual”, "de usuarios". Igualando los atributos de clase social, sexo y sector institucional 
Tabla 3. Estimación de modelos jerárquico lineales (HLM) alternativos de desigualdad educativa para PISA 2009. Coeficientes de regresión (efectos fijos) y efectos aleatorios (varianzas).

\begin{tabular}{|c|c|c|c|c|c|}
\hline & $\begin{array}{l}\text { Modelo } \\
\text { Frontera }\end{array}$ & $\begin{array}{l}\text { Modelo } \\
\text { estándar }\end{array}$ & $\begin{array}{c}\text { Modelo } \\
\text { estándar y } \\
\text { frontera }\end{array}$ & $\begin{array}{l}\text { Modelo } \\
\text { portuñol }\end{array}$ & $\begin{array}{l}\text { Modelo } \\
\text { compos. }\end{array}$ \\
\hline Constante & $411.4 * *$ & $400.2 * *$ & $400.5^{* *}$ & $402.0 * *$ & $404.5 * *$ \\
\hline Entorno sociocultural escuela & & $59.1 * *$ & $58.2 * *$ & $58.3 * *$ & $58.3^{* *}$ \\
\hline Comp. Ling. Escuela & & & & 0.0 & -17.6 \\
\hline Sector Privado & & 1.2 & 2.0 & 1.8 & 1.6 \\
\hline Sector Técnica & & -15.8 & -16.1 & -15.7 & -15.1 \\
\hline Zona del portuñol & $-56.1 * *$ & & -8.5 & -3.6 & 4.4 \\
\hline $\begin{array}{l}\text { Estatus socio económico y } \\
\text { cultural estudiante }\end{array}$ & & $15.8^{* *}$ & $15.8^{* *}$ & $16.0^{* *}$ & $16.0^{* *}$ \\
\hline Es mujer & & $38.6^{* *}$ & $38.6^{* *}$ & $38.6^{* *}$ & $38.6^{* *}$ \\
\hline Es usuario del portuñol & & & & $-10.2 * *$ & $-9.8 * *$ \\
\hline \multicolumn{6}{|l|}{$\begin{array}{l}\text { Efectos Aleatorios } \\
\text { (Varianzas) }\end{array}$} \\
\hline $\operatorname{Var}(\mathrm{U} 0)$ & 4578.7 & 1040.8 & 1046.5 & 1041.7 & 1046.0 \\
\hline $\operatorname{Var}(\mathrm{r})$ & 5834.1 & 5270.9 & 5270.2 & 5258.9 & 5258.2 \\
\hline Total variance (Yij) & 10412.8 & 6311.7 & 6316.7 & 6300.7 & 6304.1 \\
\hline $\begin{array}{l}\text { Pseudo R2 Snidjers \& } \\
\text { Boskers (2012 eq. 7.3) }\end{array}$ & $100.0 \%$ & $60.6 \%$ & $60.7 \%$ & $60.5 \%$ & $60.5 \%$ \\
\hline
\end{tabular}

Fuente: Elaboración propia con base en los microtados Pisa 2009 de Uruguay.

al que asiste, un estudiante a los 15 años que se autodientifique como hablante del portuñol tiene en promedio 10 puntos menos (más de dos desvíos estándares menos) que un estudiante monolingüe español. La diferencia no estaría estadísticamente relacionada ni con el área geográfica de la frontera, ni con la delimitación más restringida del área del portuñol. En consecuencia y desde el punto de vista del enfoque de la desigualdad desarrollado en otros trabajos (Fernández, 2010; Fernández, Cardozo, 2011), el resultado avala una tesis de desigualdad por estratificación sociolingüística, pero no una tesis de segmentación de áreas geográficas.

Este hallazgo preliminar tiene algunas limitaciones que son necesarias explicitar e implicancias para la agenda de investigación. Comenzando por las primeras, deben considerarse al menos dos, las cuales presentamos a continuación, conjuntamente con posibles líneas de investigación a continuar. 
En primer lugar, el indicador de hablante de portuñol utilizado aquí tiene distintas debilidades metodológicas. Sería importante estudiar si los efectos observados son robustos a una identificación más restringida y educativamente más pertinente de "usuario del portuñol", tal como podría ser el ámbito del centro educativo (recreos y aula de clases). Es posible discutir que el problema estaría localizado principalmente entre los estudiantes que no han podido interactuar en español en el espacio formal de una escuela que ha sido monolingüe y además espacio de legitimación por excelencia del "idioma nacional". Otro aspecto clave sería estudiar una aproximación diferente a la metodología de la autoidentificación que permita corregir la estimación de usuarios incorporando otros indicadores indirectos que permitan identificar mejor la población de hablantes de portuñol. Ambos aspectos podrían ser parte de un futuro trabajo que explore estos resultados.

En segundo lugar, es necesario establecer que los resultados son válidos para la muestra de Pisa, pero no para la cohorte de edad, esto es para la población nacida entre el 1 de abril de 1993 y el 31 de marzo de 1994. Según lo que publica el Programa Pisa Uruguay (Peri, 2010), el 82\% de la cohorte de edad estaba escolarizada a nivel nacional, en tanto que se puede estimar que el $76.5 \%$ lo estaba en el área de la frontera. Se puede cuestionar si este $23.5 \%$ que no estaba asistiendo en 2009 y que es posible hipotetizar con base en antecedentes como joven desafiliado del sistema educativo, con mayor probabilidad sea un hablante del portuñol, monolingüe incluso, que un hablante sólo del español. Esto es, que la estimación general de la desigualdad esté subestimada por un sesgo de selección. De ser así, un examen avanzado de la desigualdad lingüística en los aprendizajes debería encaminarse hacia el análisis de la etapa de la Primaria (o incluso de la primar transición entre la familia y la educación inicial) y luego estimar la incidencia del factor en el tránsito a la Educación Media y en la desafiliación.

Finalmente, este primer estudio tiene algunas implicancias claras en términos de políticas educativas. Sólo queremos proponer dos. La primera y más notoria es que más allá de los ensayos que puedan hacerse para mejorar los aspectos metodológicos, existe una desigualdad lingüística que opera como factor específico y que fundamenta una política educativa específica. Esto sin dudas legitima (una vez más) la existencia y prioridad regional de un programa de educación bilingüe en la frontera, si es que el Estado asume a cabalidad el derecho a la educación sin atención al origen lingüístico, contemplado en la Constitución y que ha reconocido explícitamente en la Ley General de Educación. La segunda implicancia tiene que ver con el mismo programa bilingüe y sus diferentes componentes e implementaciones (Broveto, 2010). 
Dado que no existen medidas análogas previas al Programa, resulta difícil hipotetizar si su acción ha contribuido o no a reducir la desigualdad. Sin perjuicio de lo que pudiera informar una evaluación de impacto, resulta claro que luego de 12 años de operación en el territorio (2003), nuestro estudio reporta que las desigualdades persisten y que son importantes aún. Por lo que, la política misma debiera tener mayor atención técnica y presupuestal para que el objetivo de la igualdad esté más próximo de lo que hoy está.

\section{Referencias}

ANEP. Documentos de la Comisión de políticas lingüísticas en la educación pública. Montevideo: Administración Nacional de Educación Pública/Consejo Directivo Central, 2008.

BARRIOS, Graciela. Políticas lingüísticas en el Uruguay: estándares vs. dialectos en la región fronteriza uruguayo-brasileña. Boletim da Associação Brasileira de Linguística, n. 24, p. 65-82, 2001.

BARRIOS, Graciela; GABBIANI, Beatriz; BEHARES, Luis Ernesto; ELIZAINCÍN, Adolfo; MAZZOLINI, Susana. Planificación y políticas lingüisticas en Uruguay. Iztapalapa, v. 29, n. 13, p. 177-190, 1993.

BEHARES, Luis Ernesto; ELIZAINCÍN, Adolfo. Temas de psico y sociolingüistica. Montevideo: Facultad de Humanidades y Ciencias de la educación (UdelaR, 1981.

BEHARES, Luis Ernesto. Portugués del Uruguay y educación fronteriza. In: Cláudia Brovetto, Javier Geymonat y Nicolás Brian, Portugués del Uruguay y educación bilingüe. Montevideo: Administración Nacional de Educación Pública/Consejo de educación inicial y primaria, 2007.

BERTOLOTTI, Virginia; COLL, Magdalena. Retrato lingüístico del Uruguay: un enfoque histórico sobre las lenguas en la región. Montevideo: Comisión Sectorial de Educación Permanente, Universidad de la República, 2014.

BLANCO, Emilio. Los límites de la escuela: educación, desigualdad y aprendizajes en México. México, D. F.: El Colegio de México, 2011.

BROVETO, Claudia. Educación bilingüe de frontera y políticas lingüísticas en Uruguay. Pro-Posições, v. 21, n. 3, p. 25-43, $2010<10.1590 /$ S0103-73072010000300003>.

CÁNEPA, Gustavo; CARREÑO, Graciela. Regionalización del Uruguay en clave de Educación Superior. Montevideo: Universidad de la República/CCI, 2011.

CERVINI, Rubén. Desigualdades en el logro académico y reproducción cultural en Argentina. Revista Mexicana de Investigación Educativa, v. 7, n. 16, p. 445-500, 2002.

CERVINI, Rubén; BASUALDO, Marisa. La eficacia del sector público: el caso de las escuelas secundarias técnicas en Argentina. Revista Latinoamericana de Estudios Educativos, v. 33, n. 3, p. 53-92, 2003. 
ELIZAINCÍN, Adolfo. Historia del español en el Uruguay. Montevideo: Facultad de Humanidades y Ciencias de la educación, 1992 [In: C. Hernández (org.). Historia y presente del español en América. Madrid, Junta de Castilla y León \& Pabecal, 1992. p. 743-758].

ELIZAINCÍN, Adolfo; BEHARES, Luis Ernesto; BARRIOS, Graciela. Nos falemo brasilero: dialectos portugueses en Uruguay. Montevideo: Amesur, 1987.

FERNÁNDEZ, Tabaré. La desigualdad educativa en Uruguay entre 1996 y 1999. Revista electrónica iberoamericana sobre calidad, eficacia y cambio en educación, V. 1, n. 1, 2003.

FERNÁNDEZ, Tabaré. Distribución del conocimiento escolar: clases sociales, escuela y sistema educativo en América Latina. México, D. F.: El Colegio de México, 2007a.

FERNÁNDEZ, Tabaré. Persistent inequalities in Uruguayan primary education 19962002. In Richard Teese; Stephen Lamb; Marie Duru-Bellat. International studies in educational inequalitiy, theory and policy. v. 2: Inequality in education systems. Dordrecht: Spinger, 2007b. p. 177-202.

FERNÁNDEZ, Tabaré. La desigualdad de aprendizajes en México 1995-2006. In: Fernando Cortés; Orlandina de Oliveira (orgs.). Los grandes problemas de México: desigualdad social. t. 5. México, D. F.: El Colegio de México, 2010. p. 235-308.

FERNÁNDEZ, Tabaré; CARDOZO, Santiago. Tipos de desigualdad educativa, regímenes de bienestar e instituciones en América Latina: un abordaje con base en Pisa 2009. Páginas de Educación, v. 4, n. 1, p. 33-55, 2011.

FERNÁNDEZ, Tabaré; ARMÚA, Marcela; BERNADOU, Olga; CENTANINO, Ivanna; FERNÁNDEZ, Marlene; LEYMONIÉ, Julia; ROSSELLI, Anna; SANCHEZ, Helvecia. Uruguay en Pisa 2006: primeros resultados en ciencias, matemática y lectura del Programa internacional de evaluación de estudiantes. Montevideo: Administración Nacional de Educación Pública, 2007.

GONZALBO, Pilar. Educación rural e indígena en Iberoamérica. México, D. F.: El Colegio de México, Universidad Nacional de Educación a Distancia, 1999.

ORTIZ, Luis. Bilingüismo y educación: la diferenciación social de la lengua escolar. América Latina Hoy, v. 60, p. 139-150, 2012.

PARKER, Susan; RUBALCAVA, Luis; TERUEL, Graciela. Language barriers and school inequalities of the Indigenous in Mexico. In: Jere Berhman; Alejandro Gaviria; Miguel Székely. Who's in and who's out: social exclusion in Latin America. Washington, D. C.: Inter-American Development Bank, 2003. p. 145-177.

PÉREZ, Elías. La crisis de la educación indígena en el área tzotzil. Los Altos de Chiapas. México, D. F.: Universidad Pedagógica Nacional, 2003.

PERI, Andrés. Primer informe Uruguay en Pisa 2009. Montevideo: Administración Nacional de Educación Pública (Anep), 2010.

RAUDENBUSH, Stephen; BRYK, Anthony. Hierarchical linear models. Thousand Oaks, CA: Sage, 2002.

RONA, José Pedro. El dialecto "fronterizo" del norte del Uruguay. Montevideo: Facultad de Humanidades, Universidad de la República, 1965. 
STAVENHAGEN, Rodolfo. Derecho indigena y derechos humanos en América Latina. México, D. F.: El Colegio de México, 1998.

Recebido em: 5 out. 2015

Aprovado em: 14 mar. 2016

Autor correspondente:

Tabaré Fernández Aguerre

Departamento de Sociología, Universidad de la República

Avda. Constituyentes 1502, Piso 5. Oficina 518

Montevideo 11.100. Uruguay 